\title{
Qualität als Kriterium der Krankenhausplanung
}

\section{Gruhl}

Qualitätsmanagement

Schlüsselwörter

- Krankenhausplan

Krankenhausplanung

Qualitätsindikatoren

Qualitätsmonitoring

Key words

hospital-plan

hospital-planning

quality indicators

quality monitoring
Institut

Abteilung Gesundheitswesen bei der Senatorin für Arbeit, Frauen, Gesundheit, Jugend und Soziales

Bibliografie

DOI $10.1055 / \mathrm{s}-0028-1085600$

Dtsch Med Wochenschr 2008;

133: S156 - (c) Georg Thieme

Verlag KG Stuttgart - New York . ISSN 0012-0472

Korrespondenz

Dr. Matthias Gruhl

Abteilungsleiter

Gesundheitswesen

Bei der Senatorin für Arbeit,

Frauen, Gesundheit, Jugend

und Soziales

Bahnhofsplatz 29

28195 Bremen

Tel. +49 (421) 3619328

eMail matthias.gruhl@

gesundheit.bremen.de
Die Länder nehmen ihren gesetzlichen Sicherstellungsauftrag für die stationäre Versorgung mittels der Krankenhausbedarfsplanung wahr. Durch die Festlegung der für die Leistungsgewährung der gesetzlichen Krankenkassen verbindlichen Planbetten/Planzahlen sowie durch die Finanzierung von notwendigen Krankenhausinvestitionen konnten die Länder bisher die Verfügbarkeit von notwendigen stationären Leistungen sicherstellen und durchsetzen.

Im Sinne einer höheren wettbewerblichen Vertragsfreiheit zwischen Kostenträgern und Krankenhäusern haben die Länder in der Gesundheitsministerkonferenz 2007 ihre Bereitschaft erklärt, auf eine bettengenaue Krankenhausplanung zu Gunsten einer gröberen Rahmenplanung zu verzichten. Der Staat nimmt sich hier bewusst zurück. Positive Erfahrungen mit einem solchen Vorgehen gibt es auf der Grundlage freiwilliger Selbstverpflichtungen bereits in mehreren Ländern.

Ein weiterer Schritt zu mehr Eigenverantwortlichkeit der Krankenhäuser kann durch die Übertragung von Investitionsmitteln in die Verfügungsgewalt der einzelnen Krankenhäuser mittels Investitionspauschalen erfolgen. Diesen Weg ist Nordrhein-Westfalen bereits gegangen, andere Länder werden in Kürze folgen, sobald sich der ordnungspolitische Rahmen auf Bundesebene geklärt hat. Auch das Bundesministerium für Gesundheit hat Sympathie für diese neue Art der betriebswirtschaftlich sinnvollen Finanzierung.

Allerdings verlieren die Länder mit diesen veränderten Tendenzen der Krankenhausplanung und Finanzierung ihre wichtigsten Instrumente zur Durchsetzung einer für notwendig erachteten Bedarfsplanung. Es besteht insofern für sie die Notwendigkeit, andere Kriterien zur Gewährleistung ihres Sicherstellungsauftrages $\mathrm{zu}$ entwickeln. Bei der Diskussion des ordnungspolitischen Rahmens wurde deshalb von der Gesundheitsministerkonferenz 2007 vorgegeben, dass die Krankenhausplanung stärker die Kriterien der Qualität berücksichtigen soll. Krankenhausplanung soll in Richtung einer Qualitätsplanung umorientiert werden. Damit wird die Erfüllungsverantwortung, die die Länder bisher für die Umsetzung der Krankenhausplanung hatten, in eine Gewährleistungsverantwortung für gute Qualität in der stationären Versorgung verändert [1]. So soll nicht die bloße Vorhaltung von Betten zu einer Vergütung von Leistungen führen, sondern die Qualität der Leistungen als Kriterium herangezogen werden.
Zwei denkbare Ansatzpunkte für eine solche Qualitätsplanung können dabei entwickelt werden: 1. Die Gewährleistung einer am Facharztstandard ausgerichteten Strukturqualität als Voraussetzung zur Aufnahme in den Krankenhausplan.

2. Die Erfüllung guter Prozess- und Ergebnisqualitäten im Sinne eines Qualitätsmonitorings als Bestandsvoraussetzung im Krankenhausplan [2].

Für die Strukturqualität liegen notwendige Indikatorensysteme bereits über die Weiterbildungsordnungen der Ärztekammern vor. Auch haben anderen Staaten (z.B. Österreich) ausgefeilte Kataloge an Strukturqualitätsvorgaben für entsprechende staatliche Zulassungen entwickelt. Gerade die Orientierung der Krankenhausplanung an dem rechtlich abgesicherten Prinzip des Facharztstandards erscheint angesichts der in den Krankenhäusern angedachten Veränderungen der Verantwortlichkeiten unterschiedlicher Berufsgruppen von hoher Wichtigkeit.

Schwieriger gestalten sich die Definitionen guter Ergebnis- oder Prozess-Qualität, zumal sie flächendeckend für alle Disziplinen gefordert werden und für eine rechtliche Nutzung gerichtsfest formuliert sein müssen. Zwar liegen für einige Disziplinen bereits abgesicherte Indikatoren vor, ein umfassendes Qualitätsmonitoring ist jedoch noch nicht gegeben. Dabei wären vorhandene Indikatoren- und Informationssysteme zu nutzen, aber auch weiter zu entwickeln.

Mit einer solchen Qualitätsorientierung werden einige Disziplinen oder sogar Krankenhäuser als potentiell gefährdend für die Patientenversorgung erkannt, benannt und ggf. nicht mehr zur Leistungsverbringung zugelassen werden. Nur durch solche Sanktionen können die Länder ihre Verantwortung für die Sicherstellung der stationären Versorgung letztendlich gegenüber ihren Bürgerinnen und Bürgern, aber auch gegenüber den Leistungserbringern und Kostenträgern glaubhaft wahrnehmen.

Autorenerklärung: Der Autor erklärt, dass keine relevanten finanziellen Verbindungen in Bezug auf dieses Manuskript bestehen.

\section{Literatur}

1 Gruhl M. Qualitätssteuerung statt Preiswettbewerb. f\&w 2008; 3: 298-303

2 Gruhl M, Klemperer D. Nutzerkompetenz durch Qualitätstransparenz - Steuerungskriterien für das deutsche Gesundheitswesen? Gesundheit und Gesellschaft Wissenschaft (GGW) 2008; 8 (1): 7-17 